\title{
Immediate effects of active exercise with compression therapy on lower-limb lymphedema
}

\author{
Takuya Fukushima ${ }^{1,2} \cdot$ Tetsuya Tsuji $^{3}$ (D) Yufuko Sano $^{4} \cdot$ Chieko Miyata $^{3}$. \\ Michiyo Kamisako $^{4}$ - Hiroka Hohri ${ }^{4}$ - Chikako Yoshimura ${ }^{4}$ Megumi Asakura ${ }^{3}$. \\ Taro Okitsu $^{3} \cdot$ Kaori Muraoka ${ }^{3} \cdot$ Meigen Liu $^{3}$
}

Received: 13 October 2016 / Accepted: 14 March 2017 / Published online: 6 April 2017

(C) The Author(s) 2017. This article is published with open access at Springerlink.com

\begin{abstract}
Purpose Active exercise with compression therapy (AECT) is a standard treatment for gynecological cancer-related lowerlimb lymphedema (LLL) in clinical situations. However, there is insufficient evidence regarding the immediate effects of the use of AECT on LLL. The purpose of this study was to evaluate the immediate effects of AECT on LLL.

Methods Participants in this randomized controlled crossover trial comprised 23 women with LLL who completed highload AECT, low-load AECT, and compression-only therapy (CT). AECT was performed on a bicycle ergometer with short stretch bandages. Each intervention was performed for $15 \mathrm{~min}$, with successive interventions separated by a 1-week washout period. Lower-limb volume was assessed using a Perometer ${ }^{\mathrm{TM}}$ sensor (Pero-system, Wuppertal, Germany). General symptoms (pain and heaviness) and skin symptoms (pitting and stiffness) were assessed using a visual analog scale and palpation, respectively. Measurements were taken before and after each intervention. Analysis of variance using linear mixed-effect modeling was used for statistical analyses.
\end{abstract}

Tetsuya Tsuji

cxa01423@nifty.com

1 Department of Rehabilitation Medicine, Nagasaki University Hospital, Nagasaki, Japan

2 Department of Locomotive Rehabilitation Science, Unit of Rehabilitation Sciences, Nagasaki University Graduate School of Biomedical Sciences, Nagasaki, Japan

3 Department of Rehabilitation Medicine, Keio University School of Medicine, Tokyo, Japan

4 Department of Rehabilitation Medicine, Keio University Hospital, Tokyo, Japan
Results Volume decrement differed significantly between all three interventions $(P<0.05)$. Lower-limb volume was significantly reduced after high-load AECT compared to that after CT. General symptoms and skin symptoms were similar across the three interventions, but severity of pre-intervention skin symptoms correlated significantly with volume decrement after high- and low-load AECT. High-load AECT using the bicycle ergometer was more effective than CT for decreasing lower-limb volume.

Conclusions These results suggest that high-load AECT has marked effects on severe LLL.

Keywords Gynecological cancer · Lymphatic system insufficiency $\cdot$ Rehabilitation $\cdot$ Ergometer exercise

\section{Introduction}

Lymphedema is a progressive chronic condition characterized by accumulation of fluid in the tissue spaces resulting from lymphatic system insufficiency and deranged lymph transport [1]. A prevalence of $20-27.2 \%$ has been reported for secondary lower-limb lymphedema (LLL) in patients who have undergone radical interventions, including pelvic lymph node dissection, radiation therapy, and/or chemotherapy, for gynecological cancer [2-4]. LLL has negative impacts on house work [5], physical activity [5, 6], mobility [6, 7], social activities $[5,7,8]$, and psychological well-being [7]. If patients with LLL are not properly managed, the LLL will become progressively worse, causing disability in activities of daily living (ADL) [5, 7] and declines in quality of life [5, 7-9]. Prevention, early diagnosis, and early treatment are thus crucial in the management of lymphedema.

Complex decongestive physiotherapy (CDP) is widely carried out in the clinical setting as a standard treatment and 
generally involves a two-stage treatment program that comprises intensive and supportive phases [1]. The first, intensive phase consists of skin care, manual lymph drainage (MLD), multi-layered compression bandaging, and exercise with compression. The second, supportive phase consists of skin care, self-MLD, compression by an elastic garment, and continued exercise with compression to conserve the results obtained in the first phase.

Several studies have examined the effects of active exercise with compression therapy (AECT) on upper-limb lymphedema (ULL). Gautam et al. showed the effects of an 8-week home exercise program comprising resistance training and stretching on decrements in limb volume [10]. Kim et al. also reported volume decrements with an 8-week intervention that added resistance training to CDP [11]. Furthermore, Jeffs et al. revealed the effects of 26-week home-based resistance training on volume decrements [12]. Concerning immediate results, the effects of AECT comprising four 12-min sessions of exercise on lymphedema related to breast cancer [13] and of gentle arm exercise on ULL [14] have been reported. Consequently, long- and short-term AECTs are beneficial to ULL in terms of volume decrement.

On the other hand, while Katz et al. showed the feasibility of resistance training, no reports have described the long- and short-term effects of AECT on LLL [15]. We therefore focused on the immediate effects of AECT and tried to clarify appropriate modalities such as exercise intensity, timing, and type.

The purpose of this study was to evaluate the immediate effects of AECT performed using a bicycle ergometer on LLL.

\section{Methods}

\section{Study design}

This was a randomized controlled crossover trial to evaluate the immediate effects of AECT performed on a bicycle ergometer with short stretch bandages on LLL. Participants completed high-load AECT, low-load AECT, and compression-only therapy (CT) in a randomized order, with the timing of the first intervention defined as period 1 , the second one as period 2, and third one as period 3. Six patterns of intervention order were applied, with randomization in blocks of six using computer-based random number tables to eliminate any effects of order. Each intervention was performed for $15 \mathrm{~min}$, and the three interventions were each separated by a 1-week washout period to eliminate any carryover effects [14]. Measurements were taken before and after each intervention (Fig. 1).

The study protocol was approved by the ethics committee at Keio University Hospital.

\section{Participants}

Patients who had been diagnosed with secondary lymphedema were screened for eligibility and recruited by rehabilitation physicians from cancer rehabilitation or lymphedema outpatient clinics at Keio University Hospital. Informed consent was obtained from all individual participants included in the study prior to enrolment. Inclusion criteria included (1) diagnosis with gynecological cancer (stages I-IV), (2) history of treatment for gynecologic cancer, (3) age 20-80 years, and (4) ability to perform exercise on a bicycle ergometer. Exclusion criteria were (1) difficulty with communicating, (2) cellulitis, (3) leakage of lymphatic fluid, (4) deep venous thrombosis, (5) severe aneurysm, (6) severe heart disease, or (7) pulmonary embolism.

Sample size was calculated using sample size calculation software (G*Power version 3.1.9.2 for Windows; http://www. gpower.hhu.de). The sample size was based on the change in lymphedema as indicated by volume [13]. With effect size calculated from mean and standard deviation, the statistical power and statistical level of significance for the study were set at 0.8 , and 0.05 , respectively, and the sample size for each group was calculated as 13 . However, the effect size in this study seemed smaller than in the previous study, because the intervention time was shorter. In consideration of this and a potential $10 \%$ loss to follow-up, we sought to recruit 20 patients with lymphedema to provide adequate power.

\section{Outcome assessments}

Age, body mass index (BMI), cancer type, treatment, and lymphedema staging according to the International Society of Lymphology (ISL) were collected from medical records. ISL stages were defined as follows: stage I, accumulation of tissue fluid that subsides with limb elevation; stage II, limb elevation alone rarely reduces swelling and pitting is manifest; late stage II, may or may not be pitting, as tissue fibrosis is more evident; or stage III, tissue is hard and pitting is absent, with skin changes such as thickening, hyperpigmentation, increased skin folds, fat deposits, and warty overgrowths. Lower-limb volume, general symptoms, and skin symptoms were evaluated pre- and immediately post-intervention without bandaging in each intervention.

\section{Lower-limb volume}

Lower-limb volume was measured using the Perometer-type $1000 \mathrm{M}^{\mathrm{TM}}$ sensor (Pero-system, Wuppertal, Germany), following the protocol of Jeffs et al. [12]. The Perometer uses infrared light to scan images of the limb. For measurement, the patient stands with one limb inside and the other outside the frame. Infrared light transmitters located on two sides of the frame project light towards photo sensors on the opposing two 
Period1

Period2

Period3

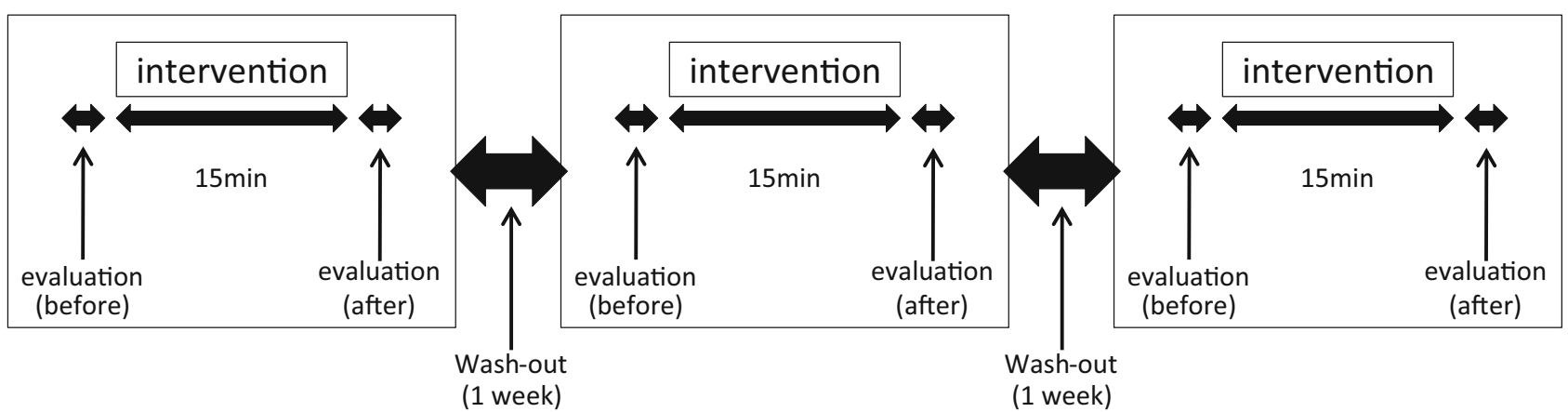

\section{Six patterns of} intervention

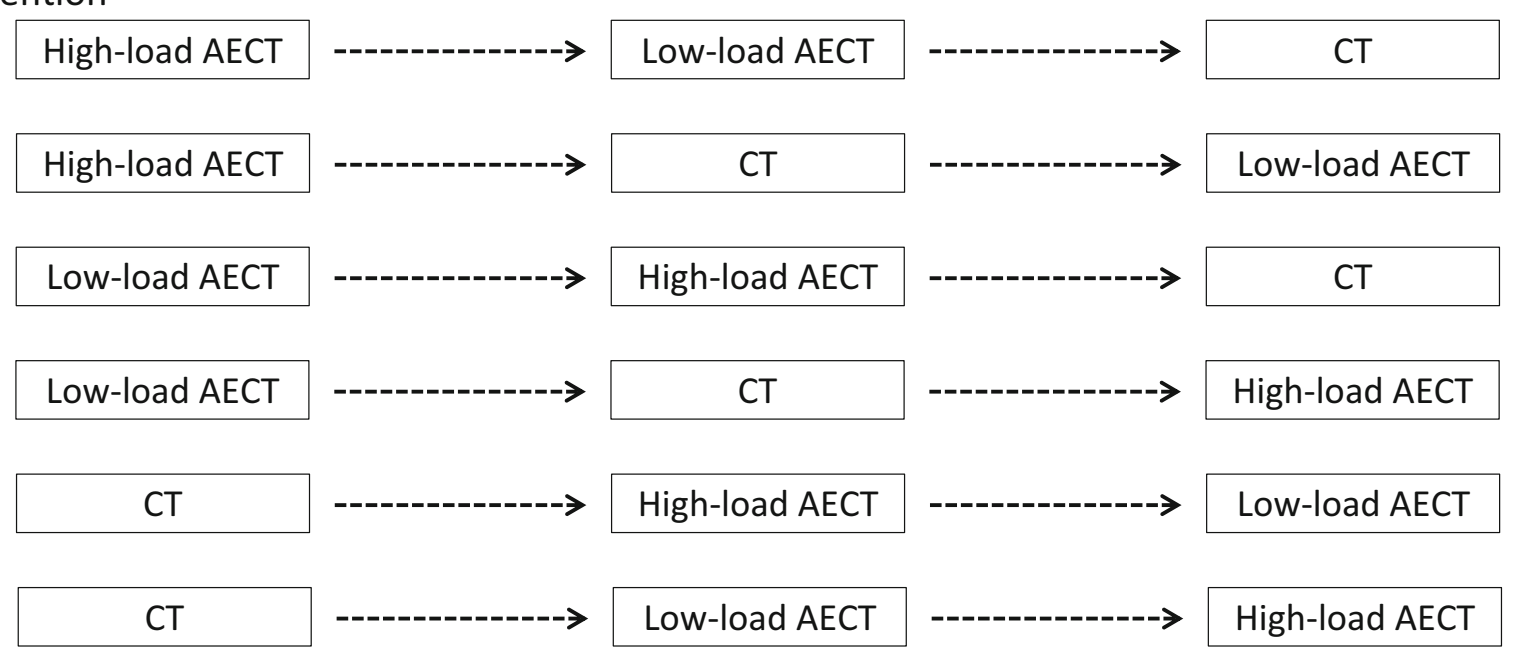

Fig. 1 Study design: the randomized controlled crossover trial. Each intervention was performed for $15 \mathrm{~min}$, and the three interventions were separated by a 1-week washout period to eliminate any carryover effects.
Measurements were taken before and after each intervention. Six patterns of intervention order were applied, with randomization in blocks of six using computer-based random number tables to eliminate order effects sites, and the limb effectively blocks transmission of the light from one side of the frame to the other, creating an electronic image. As the frame is moved along the limb, a series of images is recorded every $0.5 \mathrm{~cm}$, creating limb size and volume [16].

Measurement was performed twice and mean volume was calculated. When a difference of more than $10 \%$ was seen between the measurements, another measurement was made. After the measurement, data were uploaded to a personal computer (Lets-note ${ }^{\mathrm{TM}}$; Panasonic, Windows Vista Business, Osaka, Japan) and lower-limb volume was calculated using the PeroPlus ${ }^{\mathrm{TM}}$ software (JUZO, Cuyahoga Falls, OH).

\section{General symptoms: pain and heaviness}

Pain and heaviness were measured using a visual analog scale (VAS). On a $100-\mathrm{mm}$ horizontal line, the scale was anchored by "no pain" (score 0) and "worst imaginable pain" (score 100) for pain and "no heaviness" (score 0 ) and "worst imaginable heaviness" (score 100) for heaviness [17].
Skin symptoms: skin stiffness and pitting edema

Skin stiffness was evaluated by palpation, or pinching the skin, at four levels, defined as follows: 0 , soft; 1 , slightly hard; 2 , moderately hard; or 3, severely hard or fibrosis. Pitting edema was evaluated at three levels by palpation, pressing on the skin for $5 \mathrm{~s}$, defined as follows: 0 , pitting absent; 1 , pitting present for $\leq 5 \mathrm{~s}$; and 2 , pitting present for $>5 \mathrm{~s}$. The same physical therapist who had already received lymphedema therapist training in Japan performed the palpation. Measurements were made at medial sites $10 \mathrm{~cm}$ proximal to the knee (AK10) and $10 \mathrm{~cm}$ distal to the knee (BK10) and on the dorsal aspect of the foot. Total scores for the three sites were adopted for measurements.

\section{Maximum muscular strength of the lower limb}

Prior to the first intervention, maximum muscular strength of the lower limb was measured using the isokinetic mode of a Strength $\mathrm{Ergo}^{\mathrm{TM}} 240$ bicycle ergometer (Mitsubishi Electric 
Engineering, Tokyo, Japan). Maximum muscular strength of the lower limb was defined as the maximal peak torque, pedaling at a rate of $50 \mathrm{rpm}$. Measurements were performed twice, taking the higher value for analysis.

\section{Interventions}

All participants took part in the three interventions, comprising high-load AECT, low-load AECT, and CT in a rehabilitation room at Keio University Hospital.

In high- and low-load AECT, multi-layered compression bandaging was performed with the patient in a supine position. A foam padding bandage (Artiflex ${ }^{\circledR}$; BSN Medical, Rue Guillaume, Luxembourg) on a cotton tube stockinette (Tricofix ${ }^{\circledR}$; BSN Medical) was wrapped around the lower limb. Next, six short stretch bandages (Comprilan ${ }^{\circledR}$; BSN Medical) were sequentially wrapped around the limb with the first bandage starting at the foot, the second two bandages starting at the calf, the third bandage starting just below the knee, the fourth bandage starting at the thigh, and the fifth and sixth bandages covering the whole limb.

All bandaging was performed by the same physical therapist, who had already received lymphedema therapist training in Japan, so that sub-bandage pressure at the calf was maintained around $40 \mathrm{mmHg}$ using the Kikuhime ${ }^{\mathrm{TM}}$ pressure sensor (TT Meditrade, Sorø, Denmark), which can measure the sub-bandage pressure of bandages.

Active exercise was then performed using the training mode of the Strength $\mathrm{Ergo}^{\mathrm{TM}} 240$ bicycle ergometer. Exercise intensity was set at $10 \%$ of the maximum extension muscular strength of the lower limb as evaluated at baseline for highload AECT and 5\% for low-load AECT. Anaerobic threshold (AT) is one of the indices of exercise intensity, and energy supply is provided by aerobic mechanisms at less than AT. A previous study using the Strength $\mathrm{Ergo}^{\mathrm{TM}} 240$ bicycle ergometer showed that muscular extension strength of the lower limb required in AT was approximately $12 \%$ of the maximum extension muscular strength of the lower limb [18]. Exercise intensities were thus set at 10 and $5 \%$ of the maximum extension muscular strength of the lower limb (i.e., less than AT) so that all participants would be able to perform the exercise. The position of the seat was adjusted according to height and sitting height, so that activity of the gluteal muscles did not exceed that of the lower limbs. Participants were instructed to pedal the ergometer at 50 revolutions $/ \mathrm{min}$ for $15 \mathrm{~min}$ [17]. Exercise duration varied from 15 to $60 \mathrm{~min}$ in previous studies $[12,19,20]$. As patients with LLL may not be able to continue exercise for that long, the duration of exercise was set at $15 \mathrm{~min}$ in the present study.

During CT, participants were instructed to maintain a sitting position for $15 \mathrm{~min}$.

\section{Statistical analyses}

The Wilcoxon signed-rank test was used to analyze pre- and post-intervention changes in lower-limb volume, general symptoms, and skin symptoms. Analysis of variance using linear mixed-effect modeling was used to compare the effects of three interventions (high-load AECT, low-load AECT, and CT) and time (periods 1, 2, and 3). Least square mean (LSM) changes in lower-limb volume and percentage changes in VAS scores for general symptoms (pain and heaviness) and skin symptoms (skin stiffness and pitting edema) were used to gauge effects of the intervention. Spearman's rank correlation was used to compare the severity of pre-intervention skin symptoms (skin stiffness and pitting edema) with decrements in lower-limb volume.

Values of $P<0.05$ were considered statistically significant. Data were analyzed using the Statistics version 19 software (IBM SPSS, Chicago, IL).

\section{Results}

We enrolled 23 patients ( $25 \mathrm{limbs}$ ), of whom 22 patients (23 limbs) completed the intervention and evaluation. Two patients chose to drop out of the study before completion.

Mean age was $60.9 \pm 8.3$ years and mean BMI was $22.8 \pm 3.7 \mathrm{~kg} / \mathrm{m}^{2}$. The underlying pathology was endometrial cancer in ten patients (45.4\%), ovarian cancer in eight patients $(36.4 \%)$, and cervical cancer in four patients (18.2\%). All participants underwent surgery and half received chemotherapy. Lymphedema staging was performed according to the International Society of Lymphology (ISL) criteria, with stage II in 18 patients ( $78.3 \%$ ) and late stage II in 5 patients $(21.7 \%)$.

\section{Lower-limb volume}

Significant reductions in lower-limb volume were seen for all interventions from pre- to post-intervention (high-load AECT, $P=0.02$; low-load AECT, $P=<0.01$; CT, $P=<0.01$ ).

LSM changes in lower-limb volume differed significantly among the three interventions $(P=0.04)$, and LSM changes in lower-limb volume were significantly higher with high-load AECT than with CT $(P=0.02)$ (Table 1$)$. Although LSM changes in lower-limb volume were significantly higher for period 1 than for period $2(P=0.04)$ and period $3(P<0.01)$, no significant interactions were seen between intervention and period $(P=0.79)$.

\section{General symptoms: pain and heaviness}

Significant improvements were seen in pain (high-load AECT, $P<0.01$; low-load AECT, $P=0.01$; CT, $P<0.05$ ) and heaviness (high-load AECT, $P<0.01$; low-load AECT, 
Table 1 Mean changes in least square mean (LSM) from pre-intervention to post-intervention for lower-limb volume and general and skin symptoms

\begin{tabular}{|c|c|c|c|c|c|c|c|}
\hline \multirow[t]{2}{*}{ Outcomes } & \multicolumn{3}{|l|}{ Mean change $(95 \% \mathrm{CI})$} & \multicolumn{4}{|l|}{$P$} \\
\hline & High-load AECT & Low-load AECT & CT & $\mathrm{P} 1$ & $\mathrm{P} 2$ & $\mathrm{P} 3$ & Overall $P$ \\
\hline Lower-limb volume & $62.5 \pm 15.3(31.7-93.3)$ & $50.0 \pm 15.2(19.5-80.6)$ & $18.5 \pm 15.0(-11.6-48.6)$ & 0.02 & 0.08 & 0.48 & 0.042 \\
\hline Pain & $32.8 \pm 8.9(15.0-50.6)$ & $24.1 \pm 8.9(6.4-41.7)$ & $12.0 \pm 8.6(-5.4-29.2)$ & 0.09 & 0.31 & 0.47 & 0.22 \\
\hline Heaviness & $47.2 \pm 7.8(34.2-65.6)$ & $43.4 \pm 7.7(27.9-60.0)$ & $33.5 \pm 7.6(18.2-48.9)$ & 0.06 & 0.24 & 0.44 & 0.15 \\
\hline Skin stiffness & $2.1 \pm 1.4(-0.7-4.9)$ & $0.0 \pm 1.4(-2.8-2.8)$ & $0.0 \pm 1.4(-2.7-2.7)$ & 0.29 & 1.00 & 0.29 & 0.47 \\
\hline Pitting & $4.4 \pm 2.3(-0.2-9.1)$ & $3.7 \pm 2.3(-0.9-8.3)$ & $5.5 \pm 2.3(1.0-10.0)$ & 0.72 & 0.56 & 0.82 & 0.84 \\
\hline
\end{tabular}

Mean changes in LSM were estimated on the basis of analyses of variance using linear mixed-effect modeling. LSM changes of lower-limb volume and percentage changes in general symptoms including pain and heaviness and skin symptoms including skin stiffness and pitting edema were calculated as effects of intervention

$P 1$ high-load AECT versus CT, $P 2$ low-load AECT versus CT, $P 3$ high-load AECT versus low-load AECT, High-load AECT bicycle ergometer with compression therapy, with intensity set at $10 \%$ of maximum extension muscular strength of the lower limb, Low-load AECT bicycle ergometer with compression therapy, with intensity set at $5 \%$ of maximum extension muscular strength of the lower limb, CT compression-only therapy, $L S M$ least square mean, $C I$ confidence interval. AECT active exercise with compression therapy, $C T$ compression-only therapy

$P<0.01$; CT, $P<0.01)$ with all interventions from pre- to post-intervention.

No significant differences existed in LSM percentage changes in pain and heaviness between the three interventions $(P=0.22$ and $P=0.15)$ (Table 1$)$ and periods $(P=0.82$ and $P=0.23)$.

\section{Skin symptoms: skin stiffness and pitting edema}

Although no significant improvement of skin stiffness (highload AECT, $P=0.16$; low-load AECT, $P=1.00$; and CT, $P=1.00)$ was seen with any interventions from pre- to postintervention, a significant difference in pitting edema (highload AECT, $P=0.03$; low-load AECT, $P<0.05$; CT, $P=0.03$ ) was observed with all interventions.

No significant differences in LSM percentage change in skin stiffness or pitting between the three interventions ( $P=0.47$ and $P=0.84$ ) (Table 1$)$ and periods $(P=0.47$ and $P=0.42$ ) were observed.

Figure 2 shows that the severity of pre-intervention skin stiffness correlated significantly with changes in lower-limb volume with high-load $(r=0.66, P<0.01)$ and low-load AECT $(r=0.53, P<0.01)$, but not with CT $(r=-0.04$, $P=0.86$ ), while severity of pre-intervention pitting correlated significantly with changes in lower-limb volume under highload $(r=0.47, P=0.02)$ and low-load AECT $(r=0.45$, $P=0.03)$, but not under CT $(r=-0.31, P=0.15)$.

\section{Discussion}

No previous reports have provided data regarding the immediate effects of AECT on LLL. AECT for LLL thus lacks clear evidence regarding appropriate exercise intensity, timing, or type. Our study represents the first report of a randomized, controlled, crossover trial to evaluate the immediate effects of AECT on LLL. Changes in lower-limb volume were significantly greater with high-load AECT than with CT. Severity of skin stiffness and pitting edema symptoms preintervention correlated significantly with the volume decrement under both high- and low-load AECT.

\section{Interventions}

In our study, multi-layered compression bandaging using short stretch bandages was adopted as a compression therapy. Damstra et al. reported median sub-bandage pressure immediately after application as $64 \mathrm{mmHg}$ [21]. We applied a pressure of $40 \mathrm{mmHg}$, because a sub-bandage pressure exceeding $40 \mathrm{mmHg}$ is recommended to counteract intravenous pressure in the antigravity position [22], and sub-bandage pressure rises during exercise [18]. To reduce the unevenness of the sub-bandage pressure in each participant, measurements were made at the facies posterior cruris using Kikuhime ${ }^{\mathrm{TM}}$ pressure sensors (TT Meditrade), which provide a digital output from pressure transducers comprising an air-filled pressure sensor and the device. The accuracy and precision of Kikuhime ${ }^{\mathrm{TM}}$ have already been reported [23].

Concerning the exercise modality, we considered that it was reasonable for patients with lymphedema to perform the exercises. In our study, active exercise therapy was performed on a bicycle ergometer, which offers some benefits. First, this modality is safe because of the low risk of falls. During pedaling, muscle activity of the whole lower limb is involved [24]. In addition, pedaling reportedly places less stress on the knee joint than muscle strengthening exercises of the quadriceps with weight loading and squats [25].

This study involved a randomized crossover design in which participants completed three interventions separated by 1 -week washout periods. Moseley et al. showed that the 

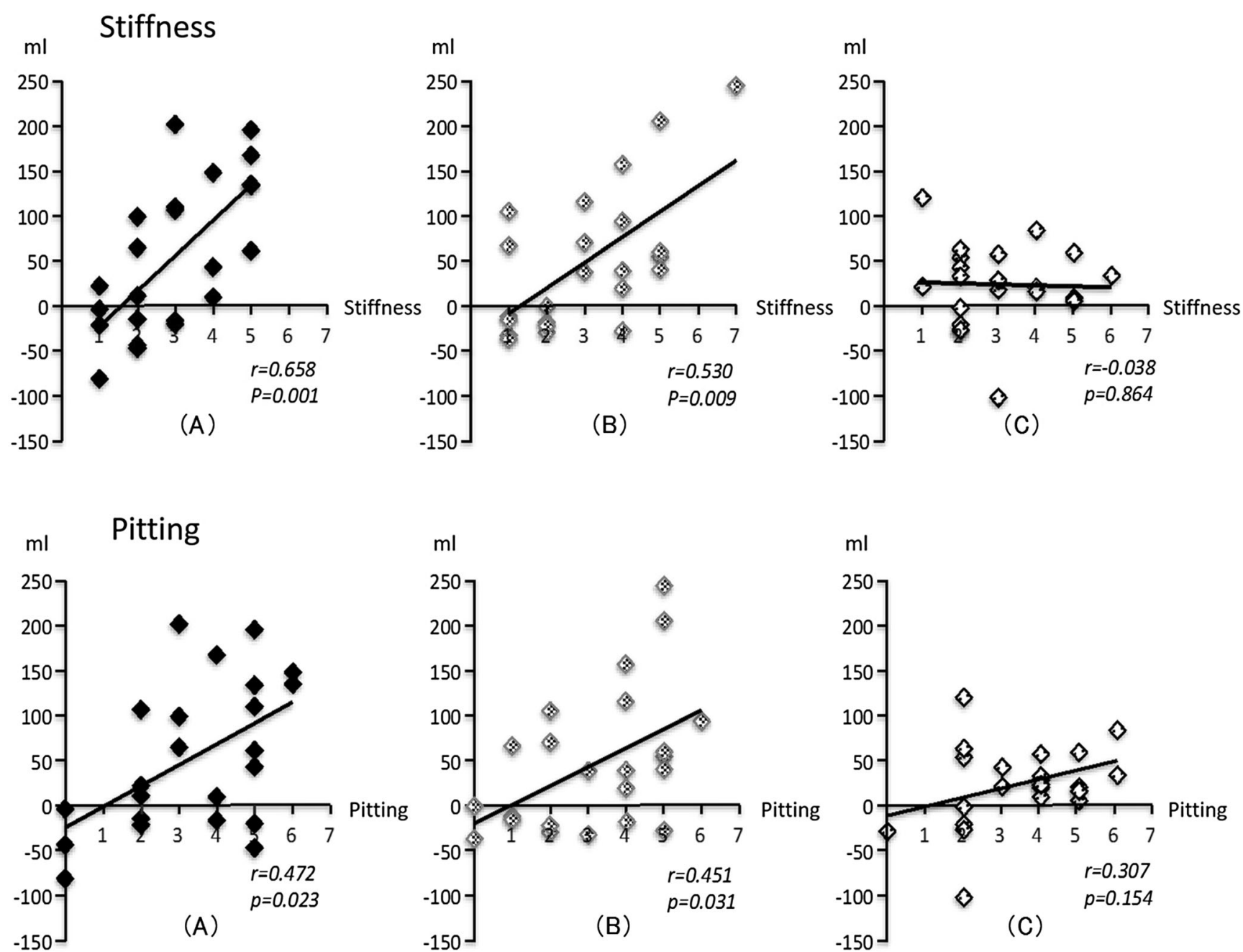

Fig. 2 Correlations between severity of pre-intervention skin symptoms and changes in lower-limb volume. Severity of pre-intervention skin stiffness correlated significantly with changes in lower-limb volume for high-load (a) $(r=0.66, P<0.01)$ and low-load AECT (b) $(r=0.53$,

$P<0.01)$, but not for CT $(\mathbf{c})(r=-0.04, P=0.86)$. Severity of preintervention pitting correlated significantly with changes in lower-limb volume for high-load (a) $(r=0.47, P=0.02)$ and low-load AECT (b) $(r=0.45, P=0.03)$, but not for CT (c) $(r=-0.31, P=0.15)$

reduction in arm volume induced by exercise gradually returned, reaching the baseline volume by $60 \mathrm{~min}$ [14]. The washout period in this study was thus judged as both necessary and sufficient.

\section{Decrement in lower-limb volume}

Our results showed that the decrement in lower-limb volume was significantly higher with AECT than with CT. Godoy et al. showed immediate effects of AECT on ULL [13], while Kim et al. [11] reported that volume reduction of the upper limb was more effectively achieved with AECT than with CT. The present study obtained similar results for LLL as the preceding studies for ULL.

CT with application of constant pressure to the skin has some beneficial effects, such as reduction of gravitational influence, restraint of excessive leakage out of blood vessels, improvement of lymph propulsion and lymphatic vessel recruitment, and increased lymphatic drainage [26]. Moreover, skeletal muscle contraction or muscle pumping in AECT can create counterpressure in the form of expansion pressure against the bandages and stimulates lymphatic flow [10-14, 27]. As a result, AECT achieves a greater volume decrement for the lower limb than CT.

Our study also detected that the decrement in lowerlimb volume was greater with high-load AECT than with low-load AECT. The higher muscle contraction may be presumed to have created and stimulated greater lymphatic flow under high-load AECT than under lowload AECT.

Concerning the period, the decrement of lower-limb volume was significantly higher in period 1 than in period 2 or 3 . Because no interaction was seen between intervention and period, and the three interventions were assigned to each 
period equally, period effect was canceled out and did not affect the results.

\section{General symptoms: pain and heaviness}

Gautam et al. showed that AECT for ULL significantly improved general symptoms [10]. This study showed similar results for LLL as the preceding studies on ULL.

In patients with lymphedema, pain is caused not only by inflammation, ischemia, complex regional pain syndrome, and cancer recurrence or progression, but also by tissue distension resulting from the accumulation of lymph fluid [27]. Reductions in lower-limb volume with AECT result in decreased lower-limb weight and tissue expansion. Although significant improvements in pain and heaviness were seen with all interventions from pre- to post-intervention, no significant differences existed between the three interventions. Because little difference in lower-limb volume decrement was evident between interventions, participants might have perceived broadly comparable levels of relief. In addition, the VAS rating scale might have been insufficient to detect differences between AECT and CT.

\section{Correlation between severity of pre-intervention skin symptoms and lower-limb volume decrement}

Because lymph contains a high concentration of plasma proteins such as albumin, the skin and subcutaneous tissue fibrosis are ongoing and harden as lymphedema becomes progressively severe [27]. Although marked pitting is often evident in ISL stage II, pitting may or may not be present as skin tissue fibrosis becomes more evident in late stage II. Furthermore, pitting is absent in ISL stage III.

Because all participants in our study were classified at stage II and late stage II and displayed some skin symptoms, significant improvement of pitting was seen with all interventions from pre- to post-intervention through the decrement in lowerlimb volume.

Although no significant difference between the three interventions was seen in pitting and skin stiffness, preintervention severity correlated significantly with the lowerlimb volume decrement in AECT. This means that AECT would be more beneficial for severe LLL. Such findings are very important in clinical practice. AECT is more effective than CT for LLL that involves more marked pitting edema and/or harder skin. On the other hand, the immediate volume decrement from AECT is comparable to that from CT in LLL with less skin symptoms. Making treatment decisions on the basis of the severity of skin symptoms may therefore be warranted.

\section{Limitations and future issues}

Use of a crossover design could reduce the influence of confounding covariates and could be statistically efficient. On the other hand, although each intervention was separated by a 1-week washout period to minimize carryover effects, the possibility of such effects cannot be completely ruled out. We used an original scale of skin symptoms based on palpation in clinical practice. Verification of psychometric properties such as reliability, validity, and reactivity is thus necessary. We also need to develop methods for quantitative evaluation.

Concerning the indication criteria, because no participants in this study were at ISL stage III, the immediate effects of $\mathrm{AECT}$ on that population remain unknown.

We examined the immediate effects of AECT, but longterm effects also need to be evaluated. In addition, exercise modalities for LLL need to be standardized by examining exercise type, intensity, duration, and frequency.

Acknowledgements This work was supported in part by JSPS KAKENHI Grant Number 26350586 and AMED Practical Research for Innovative Cancer Control Grant Number 16ck0106215. The authors thank the members of Department of Rehabilitation Medicine, Keio University School of Medicine and Keio University Hospital for their support; this research would not have been possible without their leadership and cooperation.

Compliance with ethical standards The study protocol was approved by the ethics committee at Keio University Hospital. Informed consent was obtained from all individual participants included in the study prior to enrolment.

Conflict of interest The authors declare that they have no conflicts of interest.

Open Access This article is distributed under the terms of the Creative Commons Attribution-NonCommercial 4.0 International License (http:// creativecommons.org/licenses/by-nc/4.0/), which permits any noncommercial use, distribution, and reproduction in any medium, provided you give appropriate credit to the original author(s) and the source, provide a link to the Creative Commons license, and indicate if changes were made.

\section{References}

1. International Society of Lymphology (2013) The diagnosis and treatment of peripheral lymphedema: 2013 consensus document of the International Society of Lymphology. Lymphology 46:1-11

2. Ohba Y, Todo Y, Kobayashi N, Kaneuchi M, Watari H, Takeda M, Sudo S, Kudo M, Kato H, Sakuragi N (2011) Risk factors for lowerlimb lymphedema after surgery for cervical cancer. Int J Clin Oncol 16:238-243

3. Tada H, Teramukai S, Fukushima M, Sasaki H (2009) Risk factors for lower limb lymphedema after lymph node dissection in patients with ovarian and uterine carcinoma. BMC Cancer 9:47 
4. Beesley VL, Rowlands IJ, Hayes SC, Janda M, O'Rourke P, Marquart L, Quinn MA, Spurdle AB, Obermair A, Brand A, Oehler MK, Leung Y, McQuire L, Webb PM (2015) Incidence, risk factors and estimates of a woman's risk of developing secondary lower limb lymphedema and lymphedema-specific supportive care needs in women treated for endometrial cancer. Gynecol Oncol 136:87-93

5. Dunberger G, Lindquist H, Waldenström AC, Nyberg T, Steineck G, Åvall-Lundqvist E (2013) Lower limb lymphedema in gynecological cancer survivors-effect on daily life functioning. Support Care Cancer 21:3063-3070

6. Brown JC, Lin LL, Segal S, Chu CS, Haggerty AE, Ko EM, Schmitz KH (2014) Physical activity, daily walking, and lower limb lymphedema associate with physical function among uterine cancer survivors. Support Care Cancer 22:3017-3025

7. Finnane A, Hayes SC, Obermair A, Janda M (2011) Quality of life of women with lower-limb lymphedema following gynecological cancer. Expert Rev Pharmacoecon Outcomes Res 11:287-297

8. Farrell R, Gebski V, Hacker NF (2014) Quality of life after complete lymphadenectomy for vulvar cancer: do women prefer sentinel lymph node biopsy? Int J Gynecol Cancer 24:813-819

9. Ferrandina G, Petrillo M, Mantegna G, Fuoco G, Terzano S, Venditti L, Marcellusi A, De Vincenzo R, Scambia G (2014) Evaluation of quality of life and emotional distress in endometrial cancer patients: a 2-year prospective, longitudinal study. Gynecol Oncol 133:518-525

10. Gautam AP, Maiya AG, Vidyasagar MS (2011) Effect of homebased exercise program on lymphedema and quality of life in female postmastectomy patients: pre-post intervention study. J Rehabil Res Dev 48:1261-1268

11. Kim DS, Sim YJ, Jeong HJ, Kim GC (2010) Effect of active resistive exercise on breast cancer-related lymphedema: a randomized controlled trial. Arch Phys Med Rehabil 91:1844-1848

12. Jeffs E, Wiseman T (2013) Randomised controlled trial to determine the benefit of daily home-based exercise in addition to selfcare in the management of breast cancer-related lymphoedema: a feasibility study. Support Care Cancer 21:1013-1023

13. Godoy MF, Pereira MR, Oliani AH, de Godoy JM (2012) Synergic effect of compression therapy and controlled active exercises using a facilitating device in the treatment of arm lymphedema. Int J Med Sci 9:280-284

14. Moseley AL, Piller NB, Carati CJ (2005) The effect of gentle arm exercise and deep breathing on secondary arm lymphedema. Lymphology 38:136-145

15. Katz E, Dugan NL, Cohn JC, Chu C, Smith RG, Schmitz KH (2010) Weight lifting in patients with lower-extremity lymphedema secondary to cancer: a pilot and feasibility study. Arch Phys Med Rehabil 91:1070-1076

16. Pero-System Messgeräte (2017) Pero-System Messgerte. N.p., n.d. Web. http://pero-system.de/1000M_e.htm Accessed October 10, 2015

17. McNeely ML, Campbell KL, Courneya KS, Mackey JR (2009) Effect of acute exercise on upper-limb volume in breast cancer survivors: a pilot study. Physiother Can 61:244-251

18. Partsch H, Clark M, Bassez S, Benigni JP, Becker F, Blazek V, Caprini J, Cornu-Thénard A, Hafner J, Flour M, Jünger M, Moffatt C, Neumann M (2006) Measurement of lower leg compression in vivo: recommendations for the performance of measurements of interface pressure and stiffness: consensus statement. Dermatol Surg 32:224-232 discussion 233

19. Johansson K, Hayes S, Speck RM, Schmitz KH (2013) Waterbased exercise for patients with chronic arm lymphedema: a randomized controlled pilot trial. Am J Phys Med Rehabil 92:312-319

20. Cormie P, Pumpa K, Galvão DA, Turner E, Spry N, Saunders C, Zissiadis Y, Newton RU (2013) Is it safe and efficacious for women with lymphedema secondary to breast cancer to lift heavy weights during exercise: a randomised controlled trial. J Cancer Surviv 7: 413-424

21. Damstra RJ, Brouwer ER, Partsch H (2008) Controlled, comparative study of relation between volume changes and interface pressure under short-stretch bandages in leg lymphedema patients. Dermatol Surg 34:773-778 discussion 778-779

22. Lamprou DA, Damstra RJ, Partsch H (2011) Prospective, randomized, controlled trial comparing a new two-component compression system with inelastic multicomponent compression bandages in the treatment of leg lymphedema. Dermatol Surg 37:985-991

23. Partsch H (2005) The static stiffness index: a simple method to assess the elastic property of compression material in vivo. Dermatol Surg 31:625-630

24. Hug F, Dorel S (2009) Electromyographic analysis of pedaling: a review. J Electromyogr Kinesiol 19:182-198

25. Fleming BC, Beynnon BD, Renstrom PA, Peura GD, Nichols CE, Johnson RJ (1998) The strain behavior of the anterior cruciate ligament during bicycling. An in vivo study. Am J Sports Med 26: 109-118

26. Partsch H (2012) Compression therapy: clinical and experimental evidence. Ann Vasc Dis 5:416-422

27. Lymphedema Framework (2006) Lymphedema framework best practice for the management of lymphedema. International consensus. MEP Ltd, London 